OPEN ACCESS

Edited by:

Bi-Wen Peng,

Wuhan University, China

Reviewed by:

Walace Gomes-Leal,

Federal University of Western Pará,

Brazil

Yoshinori Hayashi,

Nihon University, Japan

*Correspondence:

Xiaoping Tong

xtong@shsmu.edu.cn

Mazhong Zhang

zmzscmc@shsmu.edu.cn

Specialty section:

This article was submitted to

Non-Neuronal Cells,

a section of the journal

Frontiers in Cellular Neuroscience

Received: 15 June 2021 Accepted: 27 September 2021 Published: 08 December 2021

Citation:

Wen W, Gong X, Cheung H, Yang $Y$, Cai $M$, Zheng J, Tong $X$ and Zhang M (2021) Dexmedetomidine Alleviates Microglia-Induced Spinal Inflammation and Hyperalgesia in Neonatal Rats by Systemic Lipopolysaccharide Exposure. Front. Cell. Neurosci. 15:725267. doi: 10.3389/fncel.2021.725267

\section{Dexmedetomidine Alleviates Microglia-Induced Spinal Inflammation and Hyperalgesia in Neonatal Rats by Systemic Lipopolysaccharide Exposure}

\author{
Wen Wen ${ }^{1,2}$, Xingrui Gong ${ }^{3}$, Hoiyin Cheung ${ }^{4}$, Yanyan Yang ${ }^{1,2}$, Meihua Cai ${ }^{1,2}$, \\ Jijian Zheng ${ }^{1,2}$, Xiaoping Tong ${ }^{2,5 *}$ and Mazhong Zhang ${ }^{1,2 *}$
}

1 Department of Anesthesiology, Shanghai Children's Medical Center, Shanghai Jiao Tong University School of Medicine, Shanghai, China, ${ }^{2}$ Center for Brain Science of Shanghai Children's Medical Center, Shanghai Jiao Tong University School of Medicine, Shanghai, China, ${ }^{3}$ Department of Anesthesiology, Xiangyang Central Hospital, Hubei University of Arts and Science, Xiangyang, China, ${ }^{4}$ Department of Anesthesiology, Ruijin Hospital, Shanghai Jiao Tong University School of Medicine, Shanghai, China, ${ }^{5}$ Department of Anatomy and Physiology, Shanghai Jiao Tong University School of Medicine, Shanghai, China

Noxious stimulus and painful experience in early life can induce cognitive deficits and abnormal pain sensitivity. As a major component of the outer membrane of gram-negative bacteria, lipopolysaccharide (LPS) injection mimics clinical symptoms of bacterial infections. Spinal microglial activation and the production of pro-inflammatory cytokines have been implicated in the pathogenesis of LPS-induced hyperalgesia in neonatal rats. Dexmedetomidine (DEX) possesses potent anti-neuroinflammatory and neuroprotective properties through the inhibition of microglial activation and microglial polarization toward pro-inflammatory (M1) phenotype and has been widely used in pediatric clinical practice. However, little is known about the effects of DEX on LPSinduced spinal inflammation and hyperalgesia in neonates. Here, we investigated whether systemic LPS exposure has persistent effects on spinal inflammation and hyperalgesia in neonatal rats and explored the protective role of DEX in adverse effects caused by LPS injection. Systemic LPS injections induced acute mechanical hyperalgesia, increased levels of pro-inflammatory cytokines in serum, and short-term increased expressions of pro-inflammatory cytokines and M1 microglial markers in the spinal cord of neonatal rats. Pretreatment with DEX significantly decreased inflammation and alleviated mechanical hyperalgesia induced by LPS. The inhibition of M1 microglial polarization and microglial pro-inflammatory cytokines expression in the spinal cord may implicate its neuroprotective effect, which highlights a new therapeutic target in the treatment of infection-induced hyperalgesia in neonates and preterm infants.

Keywords: lipopolysaccharide-induced inflammation, spinal cord, hyperalgesia, microglia, dexmedetomidine 


\section{INTRODUCTION}

Neonates and preterm infants in neonatal intensive care units often undergo various invasive and painful procedures, such as intravenous cannulation, adhesive removal, fingerstick, and venous or arterial puncture (Chen et al., 2012). Noxious stimulus and painful experience in early life can induce cognitive deficits and abnormal pain sensitivity occurrence later in life (Boisse et al., 2005; Hermann et al., 2006; Wang et al., 2011; Ranger et al., 2015; Nuseir et al., 2017).

Neonates and infants also have a high incidence of infection (Rudd et al., 2020). An infection can cause hyperalgesia (Ji et al., 2016), which makes neonates and infants more sensitive and respond more exaggeratedly to painful procedures. As a major component of the outer membrane of gram-negative bacteria, lipopolysaccharide (LPS) injection mimics clinical symptoms of bacterial infections, such as systemic inflammatory responses and hyperalgesia (Wegner et al., 2014, 2015). Meanwhile, LPS injection has been reported to evoke a persistent proinflammatory reactions in the brain, which may correlate with abnormal function in the central nervous system (CNS) (Gisslen et al., 2019).

Microglia can be both detrimental and beneficial in CNS disorders (Gomes-Leal, 2012). Upon appropriate stimulation, microglia can polarize toward pro-inflammatory (M1) phenotype and anti-inflammatory (M2) phenotype. The M1 phenotype can release pro-inflammatory cytokines, while the M2 phenotype can secrete anti-inflammatory cytokines to attenuate proinflammatory responses (Marques et al., 2004; Orihuela et al., 2016). Spinal microglia play a key role in central sensitization and hyperalgesia in which the mechanism involves microglial polarization toward an M1 phenotype and the production of pro-inflammatory cytokines to facilitate pain transmission in the spinal cord (Kawasaki et al., 2008; Ji et al., 2016; Gong et al., 2017). Therefore, spinal microglial activation and inflammation have been implicated in the pathogenesis of LPS-induced hyperalgesia (Hsieh et al., 2018). Meanwhile, decreased hyperalgesia has been accompanied with the upregulation of microglial M2 phenotype and increased levels of anti-inflammatory cytokines in the spinal cord (Gong et al., 2017; Huo et al., 2018).

Neutrophils are one of the types of leukocytes in the blood. After brain damage, neutrophils can infiltrate the CNS (Carlos et al., 1997). It has been reported that neutrophils are one of the sources of pro-inflammatory cytokines and that they may contribute to CNS inflammation (Ji et al., 2007; Steinbach et al., 2013). They have also been found in the brain after systemic LPS injection (Zhou et al., 2009; Wu et al., 2020).

Dexmedetomidine, a mainly selective adrenergic $\alpha 2$ receptor agonist, has been widely used in pediatric clinical practice for sedation (Mahmoud et al., 2020). It has been reported that dexmedetomidine (DEX) displays an anti-neuroinflammatory and neuroprotective property, possibly through the inhibition of microglial activation and microglial polarization toward the M1 phenotype (Yeh et al., 2018; Gao et al., 2019). Moreover, DEX can induce M2 microglial polarization and increase levels of microglia-associated anti-inflammatory cytokines (Sun et al., 2018; Gao et al., 2019; Qiu et al., 2020), which may also implicate its anti-inflammatory effect. Nevertheless, it remains unclear whether DEX can attenuate inflammation and hyperalgesia triggered by systemic LPS injection during the neonatal period.

In this study, we examined whether systemic LPS exposure has a persistent effect on spinal inflammation and hyperalgesia in neonatal rats and further estimated the protective role of DEX in adverse effects caused by LPS injection. We found that systemic LPS injection-induced acute mechanical hyperalgesia in neonatal rats. Systemic inflammation, short-term pro-inflammatory cytokine production, and microglial activation in the spinal cord were also observed in neonatal rats that underwent LPS injection. Pretreatment with DEX significantly decreased inflammation and alleviated mechanical hyperalgesia induced by LPS. The inhibition of M1 microglial polarization and microglial pro-inflammatory cytokine expression in the spinal cord may implicate the neuroprotective effect of DEX.

\section{MATERIALS AND METHODS}

\section{Animals}

The study was approved by the Laboratory Animal Welfare and Ethics Committee of Shanghai Children's Medical Center (approval number SCMC-LAWEC-2019-0016), an affiliate of Shanghai Jiao Tong University School of Medicine, and was conducted under the guidelines of the National Institute of Health. Sprague-Dawley rats were purchased from Charles River (Beijing, China). Pregnant female Sprague-Dawley rats were obtained and kept in a room with constant temperature and humidity. All the animals were maintained on a 12-h light/dark cycle (lights on between 7:00 and 19:00 h) and had free access to food and water. After birth, rat pups were sexed, and only males were included in this study. A total of about 300 neonatal rats were used in this study.

\section{Drugs}

Lipopolysaccharide (from Escherichia coli, serotype 055: B5) was purchased from Sigma-Aldrich (United States). DEX was purchased from Yangtze River Pharmacy Group (Taizhou, China).

\section{Animal Treatment}

To explore the effects of LPS on nociceptive behavior, inflammation, and microglial activation, postnatal day 5-6 (P56) rat pups were intraperitoneally injected with saline (total volume of $0.1 \mathrm{ml}$ ) or LPS (2 $\mathrm{mg} / \mathrm{kg}$ ). The dose of LPS was determined according to previous studies (Hsieh et al., 2018, 2020). Baseline mechanical threshold and thermal latency were measured $1 \mathrm{~h}$ before saline or LPS injection. Eight, 24, and $48 \mathrm{~h}$ after LPS injection, the rat pups either underwent a nociceptive behavior test or were sacrificed for mRNA analyses, enzymelinked immunosorbent assay (ELISA), and immunofluorescence.

To explore the protective role of DEX in adverse effects caused by LPS injection, the P5-6 rat pups underwent an intraperitoneal injection of saline (total volume of $0.1 \mathrm{ml})$ or DEX $(25 \mu \mathrm{g} / \mathrm{kg})$. The dose of DEX was based on previous reports (Paeschke et al., 2017; Yeh et al., 2018). The baseline mechanical threshold was 
measured $1 \mathrm{~h}$ before saline or DEX injection. Thirty minutes after injection, the rat pups were intraperitoneally injected with saline (total volume of $0.1 \mathrm{ml}$ ) or LPS $(2 \mathrm{mg} / \mathrm{kg}$ ). They underwent a nociceptive behavior test 8,24 , and $48 \mathrm{~h}$ after the LPS injection, or were sacrificed for mRNA analyses and ELISA $8 \mathrm{~h}$ after LPS injections.

\section{Behavior Test}

The rats were placed in an acrylic box on a mesh floor $50 \mathrm{~cm}$ above the table to test for mechanical hyperalgesia. After 5 min of acclimation, von Frey filaments (North Coast Medical, United States) were used to stimulate the plantar surface of one hindpaw. Mechanical threshold was defined as the von Frey filament that evoked paw withdrawal reflex in three out of five applications (Hsieh et al., 2018).

For thermal latency, the rats were habituated to the glass surface for $5 \mathrm{~min}$ and the time for withdrawal from a heat stimulus directed at the plantar surface of the hind paw was recorded. The heat stimulus was elicited using a plantar analgesia tester (Institute of Biomedical Engineering, CAMS, China). The mean of three measures was designated as thermal latency (Gong et al., 2016).

\section{RNA Extraction and RT-qPCR}

All the animals were sacrificed in deep anesthesia with isoflurane. For total RNA extraction of tissue, the lumbar enlargement of the spinal cord was removed and homogenized with Trizol (Life Technologies, United States). The total RNA was subjected to reverse transcription using PrimeScript ${ }^{\mathrm{TM}}$ RT reagent Kit with gDNA Eraser (Takara, Japan), followed by qPCR using Green ${ }^{\mathrm{TM}}$ Premix Ex $\mathrm{Taq}^{\mathrm{TM}}$ II (Takara, Japan). For RNA extraction of sorted microglia, microglia were homogenized with Trizol (Life Technologies, United States). Then, the RNA was subjected to dissolution, denaturation, reverse transcription, and amplification as described (Chen et al., 2017). qPCR was performed using Green ${ }^{\text {TM }}$ Premix Ex Taq ${ }^{\text {TM }}$ II (Takara, Japan). The primers are shown in Table 1. Glyceraldehyde 3-phosphate dehydrogenase (GAPDH) functioned as the endogenous control gene. Messenger RNA (mRNA) expressions were analyzed according to the $2^{-\triangle \Delta C T}$ method.

\section{Enzyme-Linked Immunosorbent Assay}

The lumbar enlargement of the spinal cord and blood were collected. The blood was allowed to clot for $30 \mathrm{~min}$ at room temperature. Then, it was centrifuged at $1,000 \times g$ for $20 \mathrm{~min}$ at $4^{\circ} \mathrm{C}$. Supernatants were subsequently collected as serum. Tissues were homogenized with PBS and centrifuged at $12,000 \times g$ for $30 \mathrm{~min}$ at $4^{\circ} \mathrm{C}$. The supernatant was collected, and total protein concentration was measured using BCA Protein Assay Kit (Beyotime Biotechnology, China). Cytokine levels were determined using ELISA kits (R\&D Systems, United States). Cytokine contents were expressed as picograms of cytokines per milliliter of serum or picograms of cytokines per milligram of protein.
TABLE 1 | Primer sequences used for quantitative reverse transcription PCR (RT-qPCR).

\begin{tabular}{|c|c|}
\hline & $5^{\prime}-3^{\prime}$ \\
\hline \multirow[t]{2}{*}{ iNOS } & CAGGCTTGGGTCTTGTTAGC \\
\hline & TGTTGTTGGGCTGGGAATAG \\
\hline \multirow[t]{2}{*}{ CD86 } & AAGACATGTGTAACCTGCACC \\
\hline & AGAACCGACTIITCCGGTC \\
\hline \multirow[t]{2}{*}{$\mathrm{IL}-1 \beta$} & CTGTGACTCGTGGGATGATG \\
\hline & ACAGGGATITTGTCGTTGCT \\
\hline \multirow[t]{2}{*}{ TNF- $\alpha$} & ATCGTCTACTCCTCAGAGCC \\
\hline & ATCCAGTGAGTTCCGAAAGC \\
\hline \multirow[t]{2}{*}{ IL-4 } & CGGTATCCACGGATGTAACG \\
\hline & ACTTGTTCTTCAAGCACGGA \\
\hline \multirow[t]{2}{*}{$\mathrm{IL}-10$} & ACGCTGTCATCGATTTCTCC \\
\hline & TGTCCTGCAGTCCAGTAGAT \\
\hline \multirow[t]{2}{*}{ GAPDH } & СТСTGСTССTСССТGTTCTA \\
\hline & GGTCAATGAAGGGGTCGTTG \\
\hline
\end{tabular}

\section{Immunofluorescence}

The rats were perfused with saline followed by $4 \%$ paraformaldehyde. The lumbar enlargement of the spinal cord was removed and fixed with $4 \%$ paraformaldehyde at $4^{\circ} \mathrm{C}$, followed by $30 \%$ sucrose for cryoprotection overnight. The lumbar spinal cord sections between L4-L5 were then cut into $25-\mu \mathrm{m}$ thickness with a cryostat (Leica, Germany) and incubated with the following primary antibodies overnight: polyclonal rabbit anti-Ibal (1:500; Wako Chemistry, Japan) and polyclonal rabbit anti-MPO (1:250; Abcam, Britain). The sections were further incubated with secondary antibody goat anti-rabbit for Alexa Fluor 594 (1:1,000; Abcam, Britain) for $2 \mathrm{~h}$ at room temperature. 4',6-Diamidino-2-phenylindole (DAPI) (Beyotime Biotechnology, China) was used to stain nuclei. The sections were covered and examined using a confocal microscope (Leica, Germany).

\section{Quantitative Analysis of Microglia and Neutrophils}

For the measurement of cell numbers, a microscopic image of the spinal dorsal horn region was captured in each of the two or three sections, and the number of Ibal or MPO positively immunolabeled cells in each section was counted and averaged $\left(\right.$ cells $\left./ \mathrm{mm}^{2}\right)$. The mean value of cell counting from the spinal cord sections was used to represent one sample of the spinal cord. For the measurement of the soma size of microglia, eight Iba1 positively immunolabeled cells in each section were selected, and their soma sizes were measured and averaged. The mean value of soma sizes from spinal cord sections represents one sample of the spinal cord. The analysis of cell numbers and soma sizes was performed using the Image J software (NIH, United States), and the tissue sections were from four animals in each group.

\section{Sorting of Microglia}

The microglia were sorted by using fluorescence-activated cell sorting. The rats were perfused with PBS, and the lumbar enlargement of the spinal cord was harvested. The tissues 
were dissected into small pieces and digested with trypsin (Life Technologies, United States) for $30 \mathrm{~min}$ at $37^{\circ} \mathrm{C}$. The cell suspension was filtered and centrifuged at 1,500 rpm for $5 \mathrm{~min}$ at $4^{\circ} \mathrm{C}$. The cell pellets were washed and re-suspended in phosphate-buffered saline (PBS). Then, the cells were stained with fluorescein isothiocyanate-conjugated mouse anti-rat CD45 antibody (1:800; BD Pharmingen, United States) and allophycocyanin-conjugated mouse anti-rat CD11b antibody (1:320, BD Pharmingen, United States) for $30 \mathrm{~min}$ at $4^{\circ} \mathrm{C}$. An unstained antibody was washed in PBS. DAPI (1:10,000; Beyotime Biotechnology, China) was used to discriminate live/dead cells. The microglia were identified as $\mathrm{CD} 45^{\text {low }} \mathrm{CD} 11 \mathrm{~b}^{+}$cells and gathered with a Moflo XDP flow cytometry sorter (Beckman Coulter, United States).

\section{Statistical Analysis}

The statistical analyses were performed using Prism 8.0 (GraphPad Software, United States), and data were presented as the mean \pm SEM. The results from the behavioral test were analyzed through two-way repeated-measures ANOVA followed by Bonferroni's multiple comparisons test. The results from quantitative reverse transcription PCR (RTqPCR) were analyzed by unpaired $t$-test, Mann-Whitney test, Brown-Forsythe and Welch ANOVA test, KruskalWallis test or one-way ANOVA. The results from ELISA were analyzed by unpaired $t$-test, one-way ANOVA or Brown-Forsythe and Welch ANOVA tests. The results from immunostaining were analyzed by unpaired $t$-test. Results with $P<0.05,0.01,0.001$, or 0.0001 were considered statistically significant.

\section{RESULTS}

\section{Systemic Injection of Lipopolysaccharide Induced Acute Mechanical Hyperalgesia but Not Thermal Hyperalgesia in Neonatal Rats}

To study the effect of systemic injection of LPS on nociceptive behavior, the mechanical threshold and thermal latency were observed 8, 24, and $48 \mathrm{~h}$ after LPS injection (Figure 1A). There was no significant difference in the baseline of mechanical threshold and thermal latency between the two groups $(P>0.05)$. However, we found that rats in the LPS group showed a significant decrease in mechanical threshold 8 and $24 \mathrm{~h}$ after LPS injection compared with those in the saline group $(8 \mathrm{~h}: 0.24 \pm 0.03$ vs. $1.6 \pm 0.09$, $P<0.0001 ; 24$ h: $0.8 \pm 0.06$ vs. $1.65 \pm 0.09, P<0.0001)$ (Figure 1B). Forty-eight hours after LPS injection, there was no significant difference in mechanical threshold between rats in the LPS group and rats in the saline group $(P>0.05)$. There was no significant difference in the thermal latency between the LPS and saline groups at each time point $(P>0.05)$ (Figure 1C). In summary, LPS induced acute mechanical hyperalgesia but not thermal hyperalgesia in neonatal rats.

\section{Systemic Injection of Lipopolysaccharide Induced Increased Levels of Pro-Inflammatory Cytokines in Both Serum and Spinal Cord of Neonatal Rats}

We first examined the protein levels of pro-inflammatory cytokines interleukin-1beta (IL-1 $\beta$ ) and tumor necrosis factoralpha (TNF- $\alpha$ ) in the serum 8,24 , and $48 \mathrm{~h}$ after LPS injection. There was a dramatic increased level of IL-1 $\beta$ at 8,24 , and $48 \mathrm{~h}$ after LPS injection compared with that in the saline group ( 8 h: $4,807.71 \pm 1,027.01$ vs. $30.23 \pm 13.1, P<0.05 ; 24$ h: $325.24 \pm 66.67$ vs. $48.46 \pm 23.49, P<0.01 ; 48$ h: $101.7 \pm 19.29$ vs. $24.45 \pm 6.87, P<0.01$ ) (Figure 2A). Meanwhile, a significantly increased level of TNF- $\alpha 8 \mathrm{~h}$ after LPS injection was also observed compared with that in the saline group $(2,497.7 \pm 530.95$ vs. $44.14 \pm 9.43, P<0.05$ ) (Figure 2B).

The mRNA and protein levels of pro-inflammatory cytokines IL- $1 \beta$ and TNF- $\alpha$ in the spinal cord were also examined 8, 24, and $48 \mathrm{~h}$ after LPS injection. The rats in the LPS group showed significantly increased mRNA levels of IL-1 $\beta$ and TNF- $\alpha 8$ and $24 \mathrm{~h}$ after LPS injection compared with the rats in the saline group ( $8 \mathrm{~h}$ IL-1 $\beta$ : $74.85 \pm 26$ vs. $1.08 \pm 0.2, P<0.05$; $8 \mathrm{~h}$ TNF$\alpha: 23.72 \pm 4.94$ vs. $1.1 \pm 0.17, P<0.05 ; 24$ h IL-1 $\beta: 11.05 \pm 2.31$ vs. $1.2 \pm 0.3, P<0.05 ; 24$ h TNF- $\alpha: 16.34 \pm 3.99$ vs. $1.1 \pm 0.19$, $P<0.05$ ) (Figures 3A,B). In addition, a significant increase in the protein expression of IL- $1 \beta 8$ and $24 \mathrm{~h}$ after LPS injection was also observed in the LPS group compared with the Saline group ( 8 h: $127.97 \pm 28.11$ vs. $12.51 \pm 3.56, P<0.05 ; 24$ h: $30.9 \pm 1.02$ vs. $18.18 \pm 1.64, P<0.001$ ) (Figure $3 \mathrm{C}$ ). Meanwhile, the rats showed a significant increase in the protein expression of TNF- $\alpha$ 8 and $24 \mathrm{~h}$ after LPS injection compared with rats in the saline group ( 8 h: $4.03 \pm 0.63$ vs. $1.43 \pm 0.47, P<0.05 ; 24$ h: $3.6 \pm 0.56$ vs. $1.43 \pm 0.24, P<0.05$ ) (Figure $3 \mathrm{D})$. Forty-eight hours after LPS injection, there were no significant differences in the mRNA and protein expressions of pro-inflammatory cytokines IL-1 $\beta$ and TNF- $\alpha$ between the LPS group and the Saline group $(P>0.05)$. Taken together, short-term inflammation in the spinal cord was induced by systemic injection of LPS in neonatal rats.

\section{Systemic Injection of Lipopolysaccharide Induced Microglial Activation in the Spinal Cord of Neonatal Rats}

Upon stimulation, microglia can polarize toward the proinflammatory (M1) phenotype and release pro-inflammatory cytokines (Orihuela et al., 2016; Li J. et al., 2020). Therefore, we directly examined the mRNA expressions of M1 microglial marker-inducible NO synthase (iNOS) and CD86. It showed significantly increased expressions of iNOS and CD86 $8 \mathrm{~h}$ after LPS injection compared with that in the saline group (iNOS: $367.74 \pm 72.4$ vs. $1.59 \pm 0.82, P<0.01$; CD86: $5.8 \pm 0.94$ vs. $1.02 \pm 0.1, P<0.01$, Figures 4A,B). Meanwhile, the expression of iNOS was significantly elevated $24 \mathrm{~h}$ after LPS injection compared with that in the saline group (22.59 \pm 3.86 vs. $1.15 \pm 0.29, P<0.01$, Figure 4A), although the iNOS expression level returned back to the control level $48 \mathrm{~h}$ after LPS injection $(P>0.05)$. Besides 

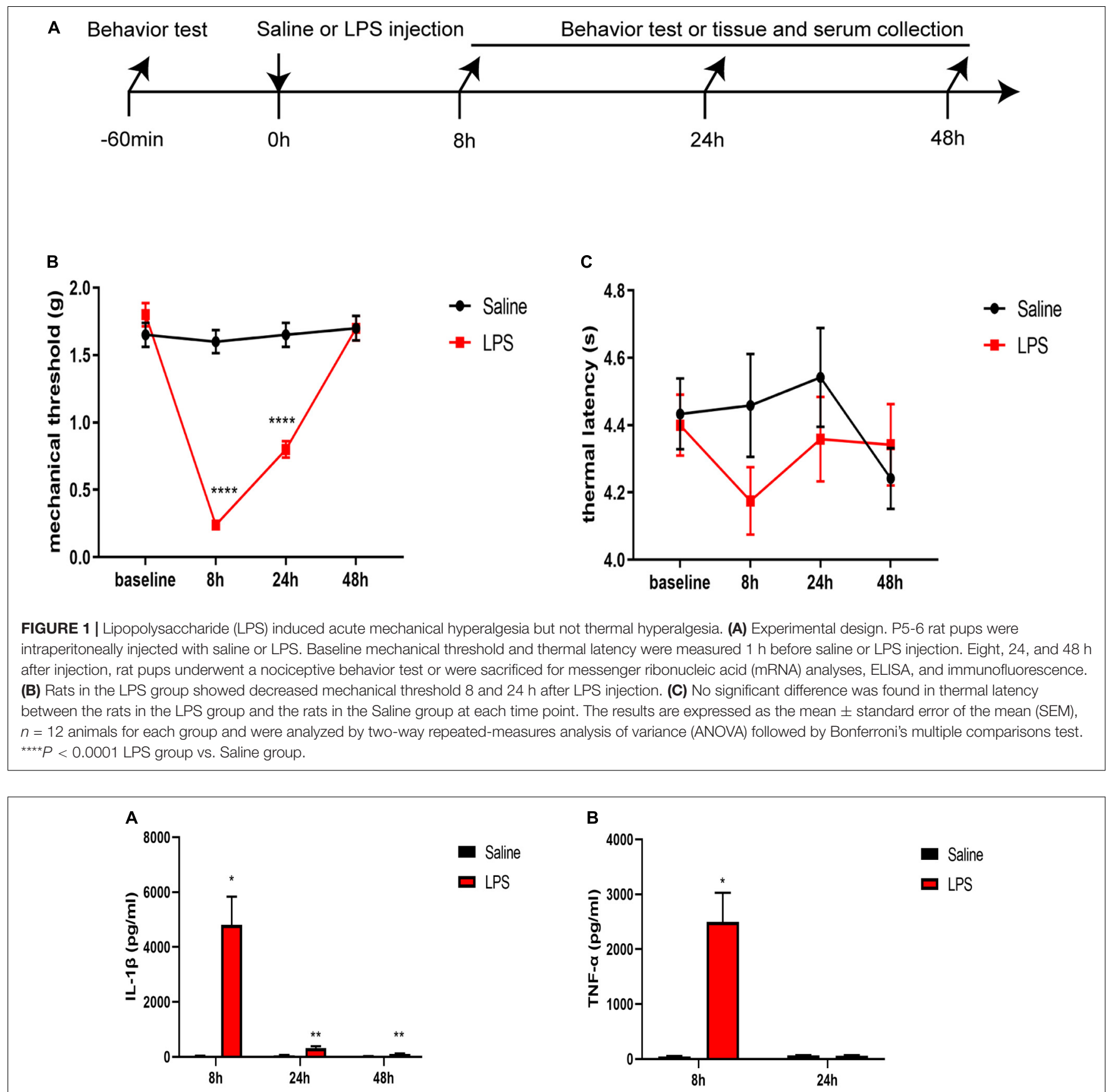

FIGURE 2 | LPS induced increased levels of pro-inflammatory cytokines in serum. (A) Serum level of IL-1 $\beta$ was elevated 8,24 , and 48 h after LPS injection in neonatal rats. (B) Serum level of TNF- $\alpha$ was elevated $8 \mathrm{~h}$ after LPS injection in neonatal rats. Results are expressed as the mean \pm SEM, $n=4-5$ animals for each group, and were analyzed by unpaired $t$-test. ${ }^{\star} P<0.05$, ${ }^{\star \star} P<0.01$ LPS group vs. Saline group.

the changes in inflammatory factors, microglia response to a noxious stimulus also includes proliferation and morphological changes (a transition from ramified to amoeboid shapes with enlarged cell bodies and shortened processes) (Chen et al., 2018). Thus, the effects of LPS exposure on microglial cell number and morphology in the dorsal horn of the spinal cord, as indicated by Ibal immunofluorescence, were determined. In short, there was no overall change in the number of Iba1-positive cells between the saline group and the LPS group 8,24 , and $48 \mathrm{~h}$ post injection $(P>0.05)$ (Figures 4C-F). However, Iba1-positive microglia showed an enlarged soma size $24 \mathrm{~h}$ after LPS injection $(124.5 \pm 9.89$ vs. $91 \pm 4.02, P<0.05$ ) (Figures $4 \mathrm{D}, \mathbf{G}$ ) but not 8 or $48 \mathrm{~h}$ post injection of LPS $(P>0.05)$ (Figures $4 \mathrm{C}, \mathbf{E}, \mathbf{G})$. Taken 
A

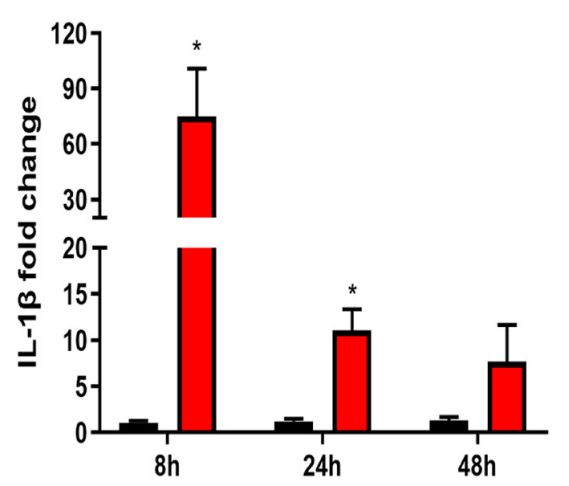

C

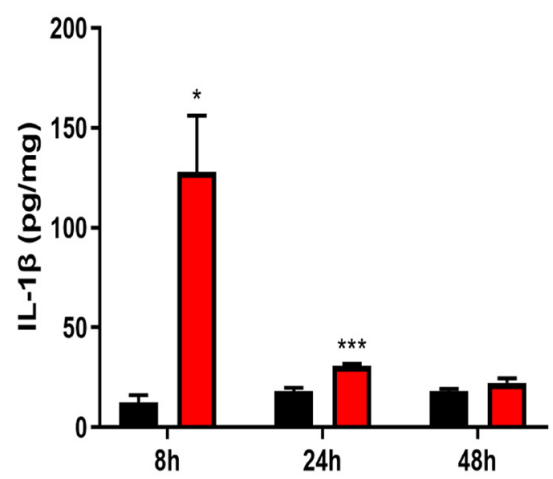

B

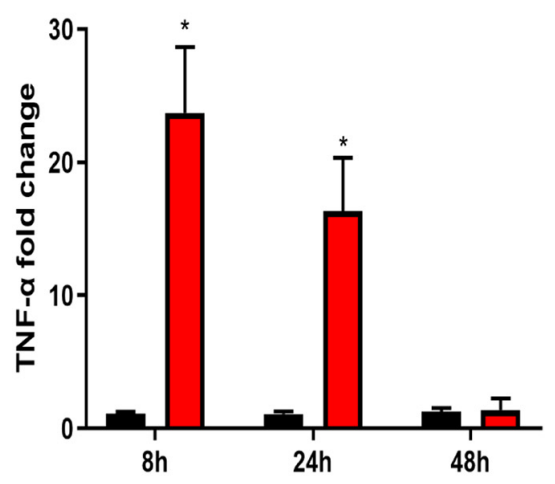

D

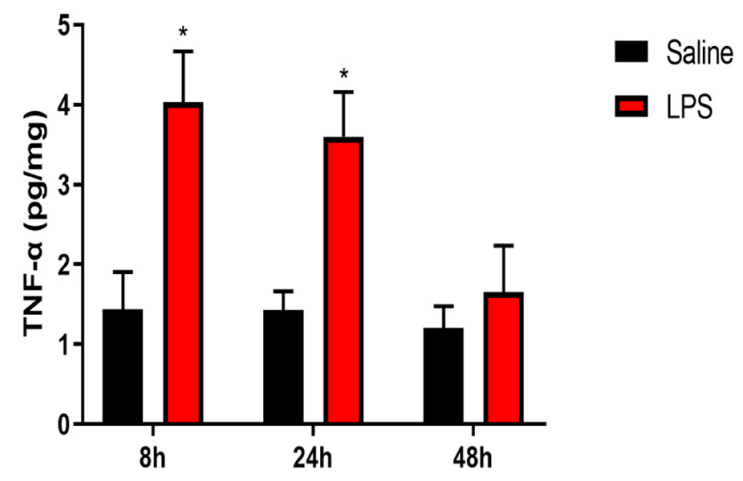

FIGURE 3 | LPS increased levels of pro-inflammatory cytokines in the spinal cord. (A,B) mRNA levels of IL-1 $\beta$ and TNF- $\alpha$ were elevated 8 and $24 \mathrm{~h}$ after LPS injection in neonatal rats. (C,D) Protein expressions of IL-1 $\beta$ and TNF- $\alpha$ were elevated 8 and $24 \mathrm{~h}$ after LPS injection in neonatal rats. Results are expressed as the mean \pm SEM, $n=4-5$ animals for each group, and were analyzed by unpaired $t$-test or Mann-Whitney test. ${ }^{\star} P<0.05,{ }^{\star \star \star} P<0.001$ LPS group vs. Saline group.

together, the results of increased mRNA expressions of M1 microglial markers and enlarged soma size in microglial morphology indicated the activation of microglia upon stimulation induced by LPS.

\section{Dexmedetomidine Pretreatment Attenuated Systemic Lipopolysaccharide-Induced Elevations of Pro-Inflammatory Cytokines and M1 Microglial Markers in the Spinal Cord of Neonatal Rats}

As DEX displays an anti-inflammatory and neuroprotective property (Li R. et al., 2020), we, thus, investigated whether DEX plays a role in the protection of neonatal rats from anti-inflammation and its possible mechanism. We found that consistent with the previous result, the rats in the Saline + LPS group showed significantly increased levels of IL- $1 \beta$ and TNF- $\alpha$ in the serum compared with the rats in the saline + saline group (IL-1 $\beta:$ 6,316.41 \pm 896.03 vs. $38.81 \pm 11.87, P<0.01 ;$ TNF- $\alpha: 1,702.62 \pm 118.48$ vs.
$36.53 \pm 11.94, P<0.001)$. However, DEX pretreatment largely decreased the serum levels of IL- $1 \beta$ and TNF- $\alpha$ (IL$1 \beta: 2,883.23 \pm 650.13$ vs. 6,316.41 \pm 896.03, $P<0.05$; TNF- $\alpha: 884.01 \pm 203.7$ vs. $1,702.62 \pm 118.48, P<0.05$, Figures 5A,B).

We further tested the DEX protective effect in the spinal cord. As shown in Figures $\mathbf{6 A , B}$, rats in the saline + LPS group showed significantly increased mRNA expressions of IL- $1 \beta$ and TNF- $\alpha$ compared with rats in the saline + saline group (IL-1 $\beta$ : $54.60 \pm 3.4$ vs. $1.05 \pm 0.12, P<0.01$; TNF$\alpha: 41.58 \pm 2.18$ vs. $1.01 \pm 0.06, P<0.001)$. In contrast, DEX pretreatment largely decreased the mRNA expressions of IL- $1 \beta$ and TNF- $\alpha$ (IL-1 $\beta$ : $5.64 \pm 2.85$ vs. $54.60 \pm 3.4$, $P<0.05$; TNF- $\alpha: 25.72 \pm 2.6$ vs. $41.58 \pm 2.18, P<0.01$ ). In addition, rats in Saline + LPS group showed significantly increased protein expressions of IL- $1 \beta$ and TNF- $\alpha$ compared with rats in the Saline + Saline group (IL-1 $\beta$ : $70.66 \pm 3.07$ vs. $11.84 \pm 4.56, P<0.0001$; TNF- $\alpha$ : $6.19 \pm 0.82$ vs. $0.96 \pm 0.25, P<0.001)$. DEX pretreatment significantly reduced the protein expressions of IL- $1 \beta$ and TNF- $\alpha$ (IL$1 \beta: 45.34 \pm 3.73$ vs. $70.66 \pm 3.07, P<0.01$; TNF- $\alpha$ : $2.43 \pm 0.66$ vs. $6.19 \pm 0.82, P<0.01$ ) (Figures 6C,D). 


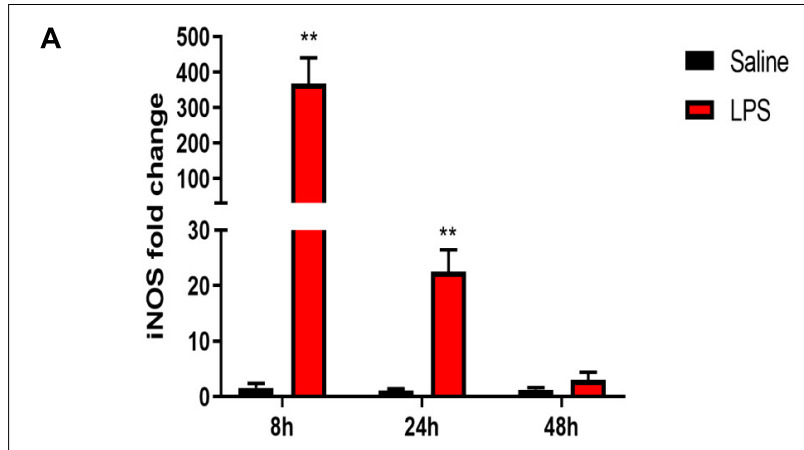

C $8 h$
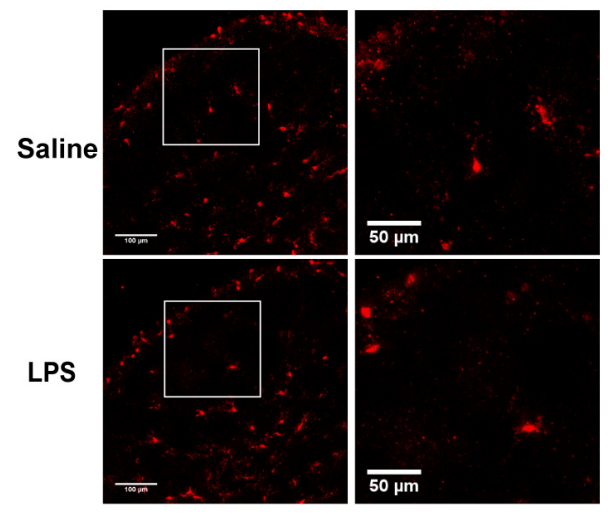

D

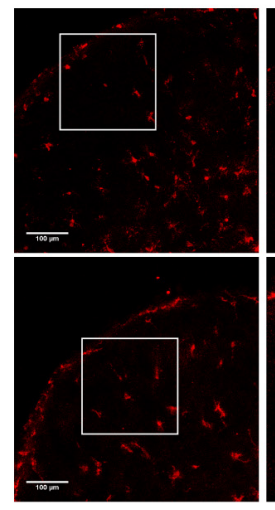

B

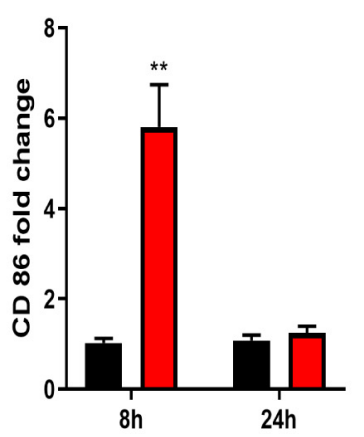

- Saline

口 LPS
E $\quad 48 \mathrm{~h}$

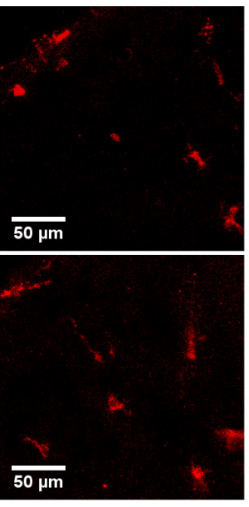

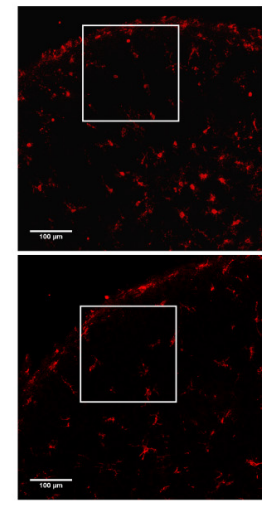

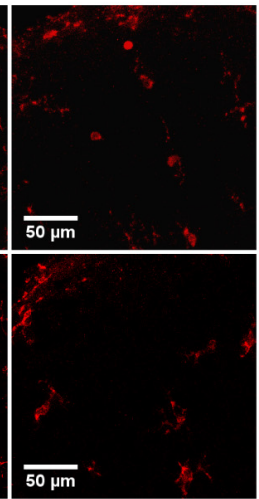

$F$

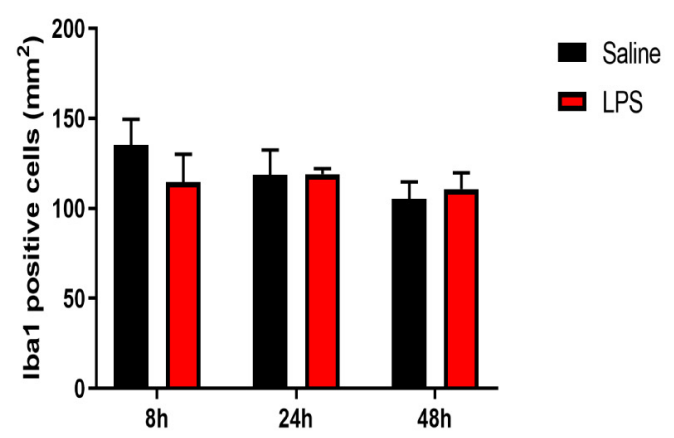

G

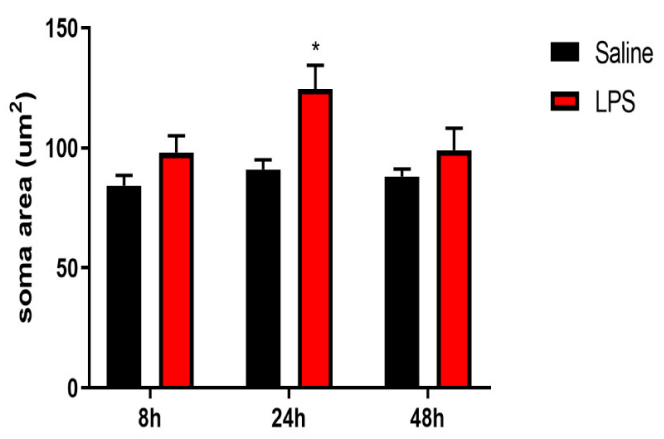

FIGURE 4 | Effects of systemic injection of LPS on M1 microglial markers, microglial number, and morphology in the spinal cord of neonatal rats. (A) mRNA level of iNOS was elevated 8 and $24 \mathrm{~h}$ after LPS injection in neonatal rats. (B) mRNA level of CD86 was elevated $8 \mathrm{~h}$ after LPS injection in neonatal rats. (C-E)

Representative photomicrographs of Iba1 immunostaining in the rat spinal cord dorsal horn 8, 24, and $48 \mathrm{~h}$ after saline or LPS injection. Scale bars, 100 and $50 \mu \mathrm{m}$ for the inset images. (F) Systemic LPS exposure did not change lba1-positive cell numbers 8, 24, and $48 \mathrm{~h}$ after LPS injection. (G) Systemic LPS exposure induced enlarged soma size of Iba1-positive microglia $24 \mathrm{~h}$ after injection. Results are expressed as the mean $\pm \mathrm{SEM}, n=4-5$ animals for each group, and were analyzed by unpaired $t$-test or Mann-Whitney test. ${ }^{*} P<0.05,{ }^{\star *} P<0.01$. LPS group vs. Saline group.

To test whether the reduction of inflammation cytokines is through the inhibition of M1 microglial polarization by DEX treatment, we found that, indeed, DEX pretreatment significantly reduced the mRNA expressions of iNOS and CD86, which are reported as M1 microglial markers (iNOS: $33.9 \pm 4.26$ vs.
$315.71 \pm 64.58, P<0.05$; CD86: $2.09 \pm 0.4$ vs. $6.32 \pm 0.64$ $P<0.0001$ ) (Figures 6E,F). Taken together, our results indicate that DEX pretreatment alleviated LPS-induced spinal inflammation, which might be through the inhibition of M1 microglial polarization. 


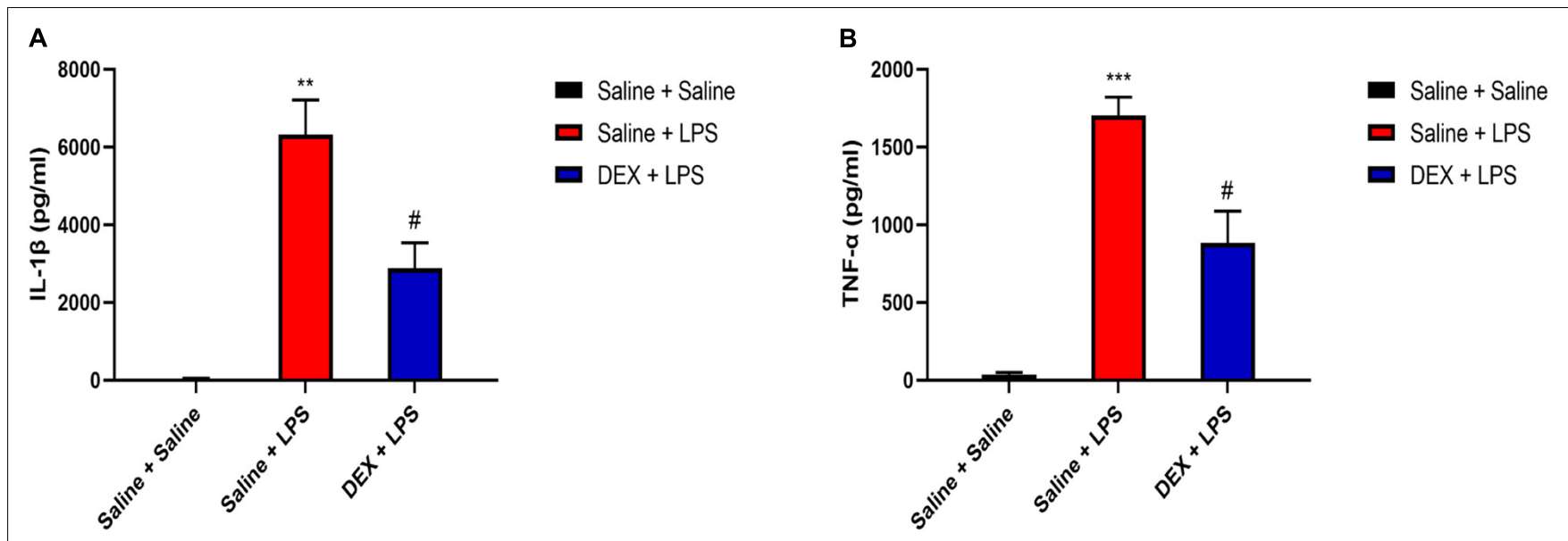

FIGURE 5 | Dexmedetomidine (DEX) pretreatment attenuated systemic LPS-induced peripheral inflammation. (A) LPS induced an increased level of IL-1 $\beta$ in serum. DEX pretreatment decreased LPS-induced upregulation of IL-1 $\beta$. (B) LPS induced an increased level of TNF- $\alpha$ in serum. DEX pretreatment decreased LPS-induced upregulation of TNF- $\alpha$. Results are expressed as the mean \pm SEM, $n=4-5$ animals for each group, and were analyzed by Brown-Forsythe and Welch ANOVA tests followed by Tamhane's T2 multiple comparisons test. ${ }^{\star \star} P<0.01$, ${ }^{\star \star \star} P<0.001$ Saline + LPS group vs. Saline + Saline group. ${ }^{\#} P<0.05$ DEX + LPS group vs. Saline + LPS group.

\section{Dexmedetomidine Pretreatment Attenuated Systemic Lipopolysaccharide-Induced Elevations of Pro-Inflammatory Cytokines in Sorted Microglia in the Spinal Cord of Neonatal Rats}

To further demonstrate the effects of DEX on the expressions of inflammatory cytokines in microglia, we assessed the mRNA expressions in sorted microglia from the lumbar enlargement of the spinal cord which the sorting strategy was shown in Figure 7A. The microglia were identified as $\mathrm{CD} 45^{\text {low }} \mathrm{CD} 11 \mathrm{~b}^{+}$cells as previously described (Zhang et al., 2002). As shown in Figures 7B,C, rats in the Saline + LPS group showed significantly increased mRNA expressions of IL-1 $\beta$ and TNF- $\alpha$ compared with rats in the Saline + Saline group (IL-1 $\beta$ : $3.55 \pm 0.25$ vs. $1.06 \pm 0.21, P<0.001$; TNF- $\alpha$ : $2.06 \pm 0.19$ vs. $1.03 \pm 0.13, P<0.01)$. In contrast, $\mathrm{DEX}$ pretreatment significantly decreased the mRNA expressions of IL- $1 \beta$ and TNF$\alpha$ (IL-1 $\beta$ : $1.93 \pm 0.32$ vs. $3.55 \pm 0.25, P<0.01$; TNF- $\alpha$ : $1.29 \pm 0.17$ vs. $2.06 \pm 0.19, P<0.05)$. As it has been reported that the anti-inflammatory effects of DEX may involve the induction of M2 microglial polarization and expressions of anti-inflammatory cytokines in microglia (Gao et al., 2019; Qiu et al., 2020), we further evaluated the mRNA expressions of anti-inflammatory cytokines interleukin-10 (IL-10) and interleukin-4 (IL-4) in sorted microglia as well. As shown in Figures 7D,E, however, we did not detect a significant difference in the expressions of IL-10 and IL- 4 between the rats in the Saline + Saline group and the rats in the Saline + LPS group $(P>0.05)$, suggesting a major role of M1 microglia in LPS-induced spinal inflammation. Meanwhile, there was no significant difference in the expressions of IL-10 and IL- 4 between the rats in the Saline + LPS group and the rats in the DEX + LPS group $(P>0.05)$ (Figures 7D,E).

\section{Dexmedetomidine Pretreatment Attenuated Systemic Lipopolysaccharide-Induced Mechanical Hyperalgesia in Neonatal Rats}

Neutrophils are important inflammatory cells that can be upregulated in the CNS after damage and are one of the sources of pro-inflammatory cytokines (Ji et al., 2007; Steinbach et al., 2013; Alam et al., 2020). We performed the immunolabeling of neutrophils with myeloperoxidase (MPO) and investigated whether the anti-inflammatory effect of DEX might be through the inhibition of neutrophil accumulation. Although we found similar enhanced immunostaining of MPO in ischemic tissue as previously reported (Kong et al., 2014) (data not shown), to our surprise, our data indicated that there was no obvious change of MPO staining in the dorsal horn of the spinal cord between the sham control and LPS-injection $8 \mathrm{~h}$ (Figures $\mathbf{8 A}, \mathbf{B}$ ), indicating that neutrophils are not the major participator in LPS-induced spinal inflammation at this point.

We next examined the effect of DEX treatment on LPSinduced mechanical hyperalgesia by the procedure shown in Figure 8C. The baseline mechanical threshold was not significantly different among the four groups $(P>0.05)$. There was no significant difference in mechanical threshold between the Saline + Saline group and the DEX + Saline group at each time point $(P>0.05)$ (Figure 8D). The rats in Saline + LPS group showed a significant decrease in mechanical threshold at 8 and $24 \mathrm{~h}$ after LPS injection compared with the rats in the Saline + Saline group ( 8 h: $0.24 \pm 0.03$ vs. $1.43 \pm 0.09$, $P<0.0001 ; 24$ h: $0.7 \pm 0.05$ vs. $1.6 \pm 0.09, P<0.0001)$. In contrast, DEX pretreatment significantly attenuated the LPSinduced mechanical hyperalgesia in neonatal rats as early as $8 \mathrm{~h}$ and had a continuous protection until the 24-h checkpoint after the LPS injection ( 8 h: $0.87 \pm 0.09$ vs. $0.24 \pm 0.03, P<0.0001$; 24 h: $1.37 \pm 0.1$ vs. $0.7 \pm 0.05, P<0.001$, Figure 8D). 
A

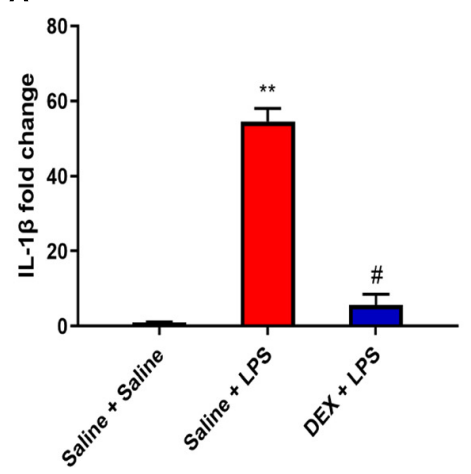

C

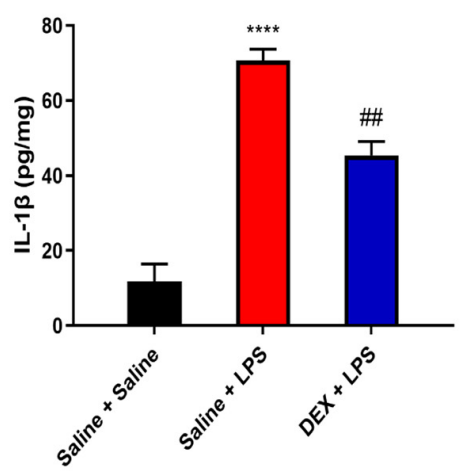

E

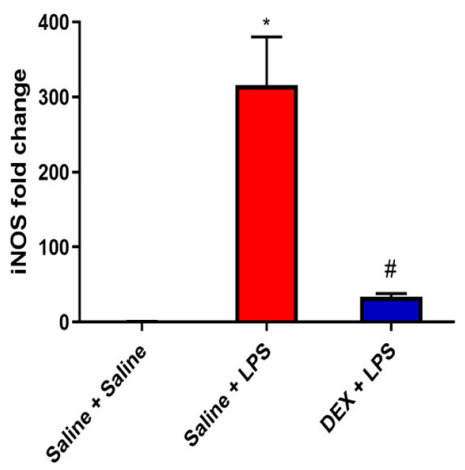

Saline + Saline

- Saline + LPS

DEX + LPS

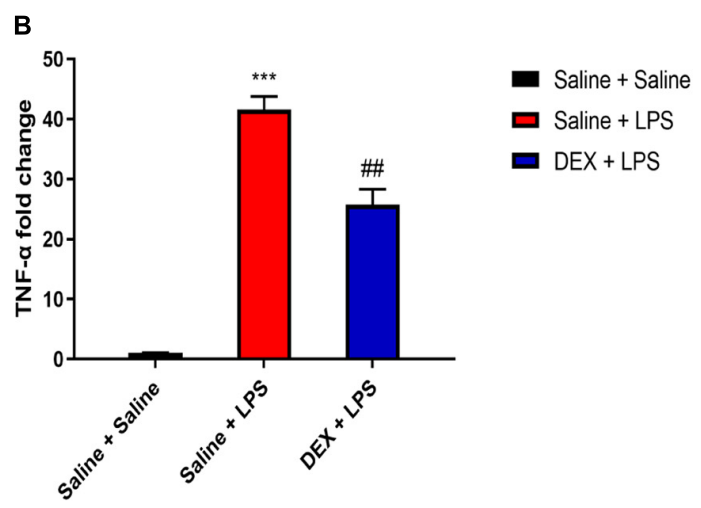

D

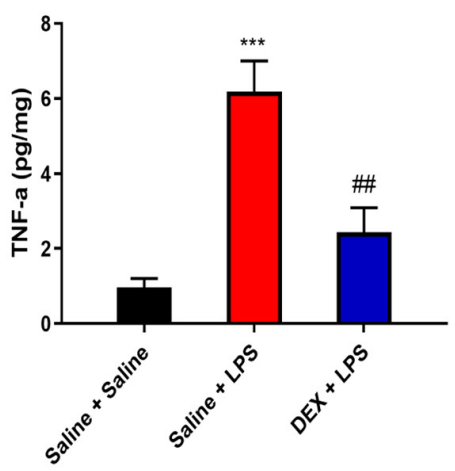

F

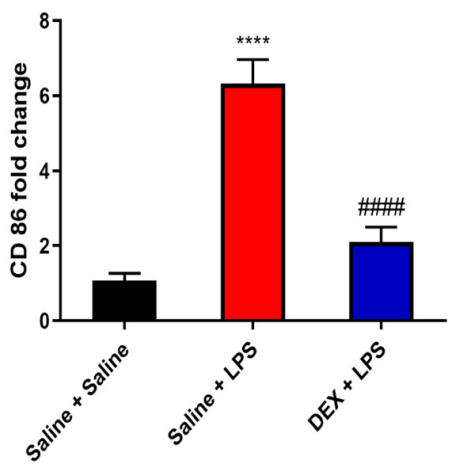

- Saline + Saline

口 Saline + LPS

- DEX + LPS
Saline + Saline

DEX + LPS

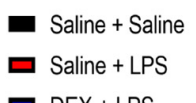

- DEX + LPS

FIGURE 6 | DEX pretreatment attenuated LPS-induced inflammation and expressions of M1 microglial markers in the spinal cord. (A,B) LPS induced increased mRNA levels of IL- $1 \beta$ and TNF- $\alpha$. DEX pretreatment decreased LPS-induced mRNA levels of IL- $1 \beta$ and TNF- $\alpha$. (C,D) LPS induced increased protein expressions of IL-1 $\beta$ and TNF- $\alpha$. DEX pretreatment decreased LPS-induced protein expressions of IL-1 $\beta$ and TNF- $\alpha$. (E,F) LPS induced increased mRNA levels of iNOS and CD86. DEX pretreatment decreased LPS-induced mRNA levels of iNOS and CD86. Results are expressed as the mean \pm SEM, $n=4-6$ animals for each group, and were analyzed by Kruskal-Wallis test followed by Dunn's multiple comparisons test, Brown-Forsythe and Welch ANOVA tests followed by Tamhane's T2 multiple comparisons test or one-way ANOVA followed by Tukey's multiple comparisons test. ${ }^{\star} P<0.05,{ }^{\star \star} P<0.01,{ }^{\star \star \star} P<0.001,{ }^{\star \star \star \star} P<0.0001$ Saline + LPS group vs. Saline + Saline group. ${ }^{\#} P<0.05,{ }^{\# \#} P<0.01,{ }^{\# \# \#} P<0.0001$ DEX + LPS group vs. Saline + LPS group.

\section{DISCUSSION}

Our study showed that a single systemic LPS injection induced acute mechanical hyperalgesia in neonatal rats. Increased levels of pro-inflammatory cytokines in serum, short-term proinflammatory cytokine production, and microglial activation in the spinal cord were also observed in neonatal rats that underwent LPS injection. Pretreatment with DEX significantly 

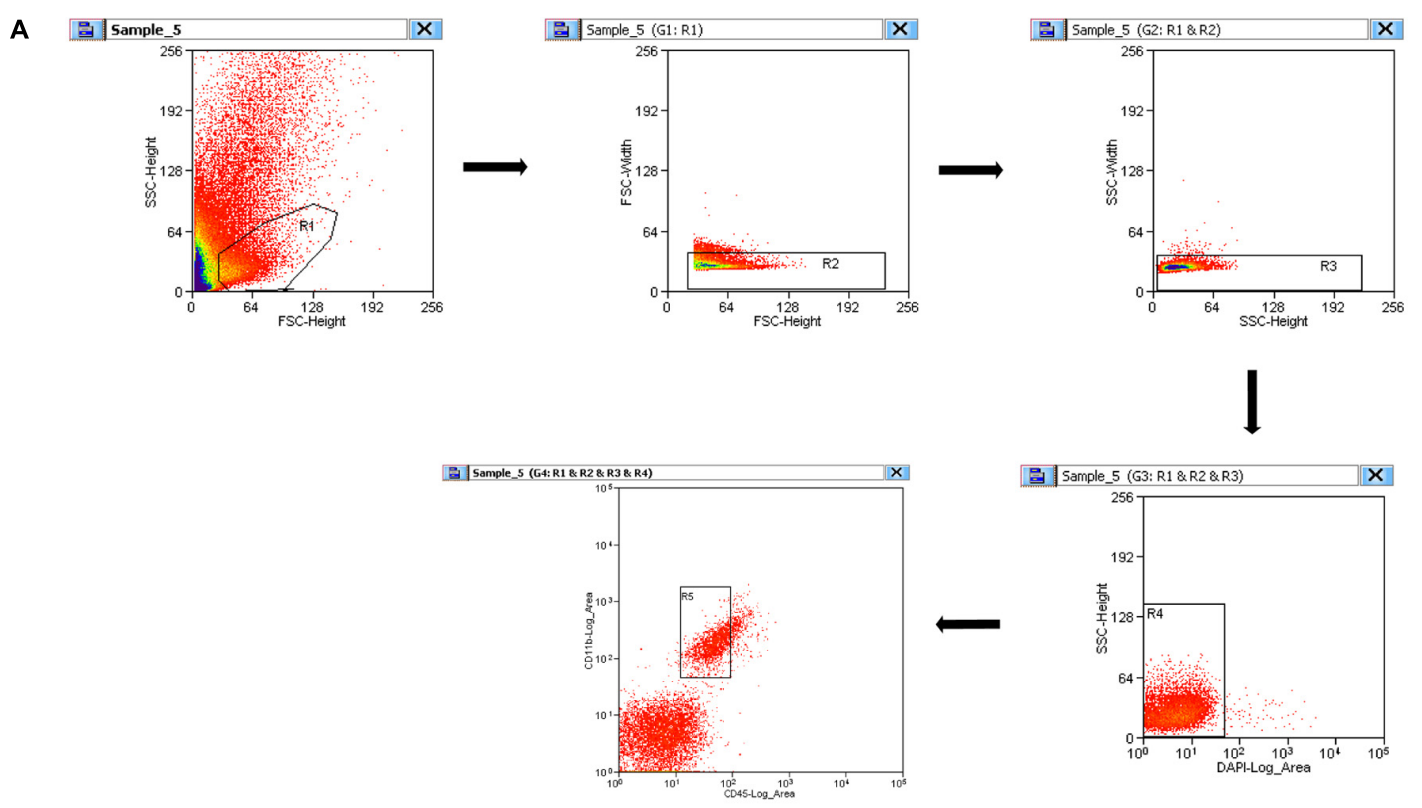

B

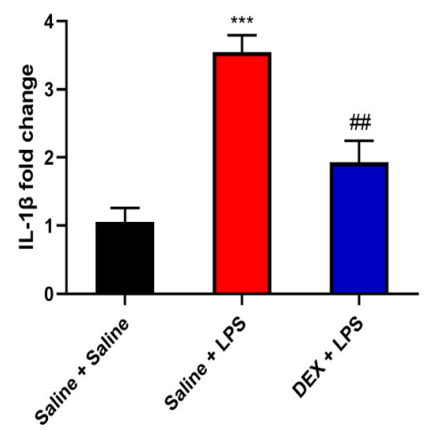

- Saline + Saline

$\square$ Saline + LPS

DEX + LPS
D

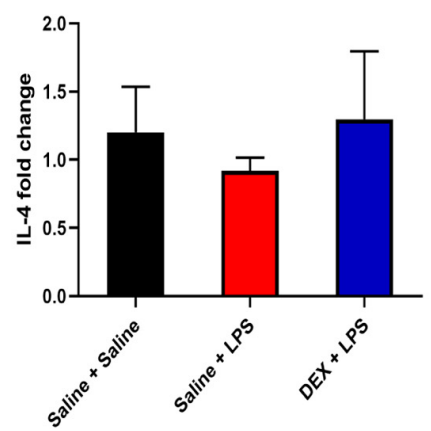

C

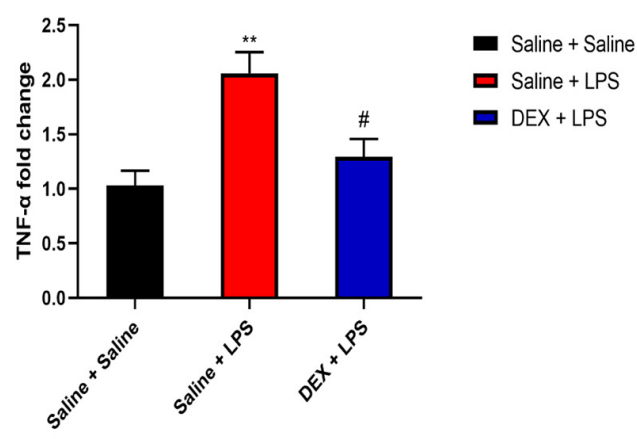

E

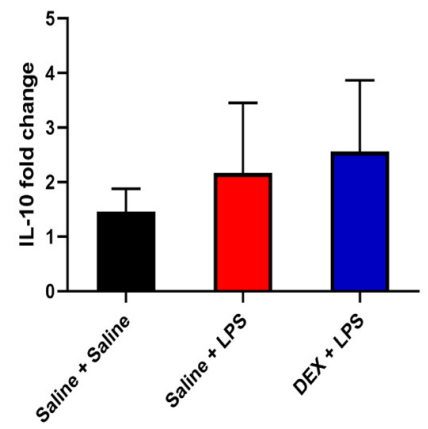

- Saline + Saline

$\square$ Saline + LPS
DEX + LPS

DEX + LPS

FIGURE 7 | DEX pretreatment attenuated the LPS-induced expressions of pro-inflammatory cytokines in microglia. (A) The representative sorting strategy of microglia from lumbar enlargement of the spinal cord in neonatal rats. Microglia were considered as CD45/ow CD11 $\mathrm{b}^{+}$cells in the R5 area and were gathered for mRNA analysis. (B,C) LPS induced increased mRNA levels of IL-1 $\beta$ and TNF- $\alpha$. DEX pretreatment decreased LPS-induced mRNA levels of IL-1 $\beta$ and TNF- $\alpha$. (D,E) Neither LPS nor DEX induced significant changes in the mRNA expressions of IL-10 and IL-4. Results are expressed as the mean \pm SEM, $n=4$ animals for each group, and were analyzed by one-way ANOVA followed by Tukey's multiple comparisons test or Kruskal-Wallis test followed by Dunn's multiple comparisons test. ${ }^{\star \star} P<0.01,{ }^{\star \star \star} P<0.001$, Saline + LPS group vs. Saline + Saline group. ${ }^{\#} P<0.05,{ }^{\# \#} P<0.01$, DEX + LPS group vs. Saline + LPS group. 
A
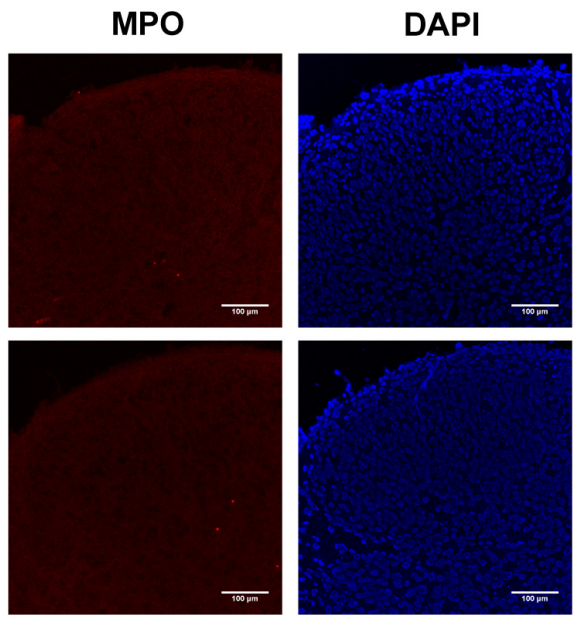

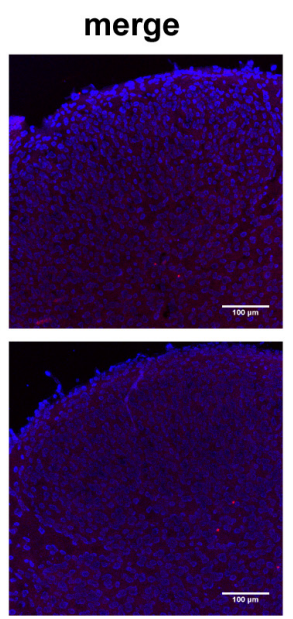

B

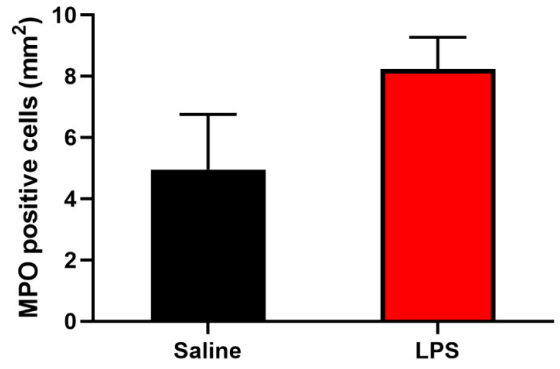

C

Behavior test

Behavior test Saline or DEX injection Saline or LPS injection Tissue and serum collection

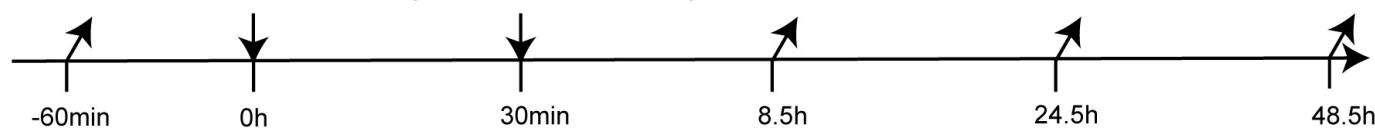

D

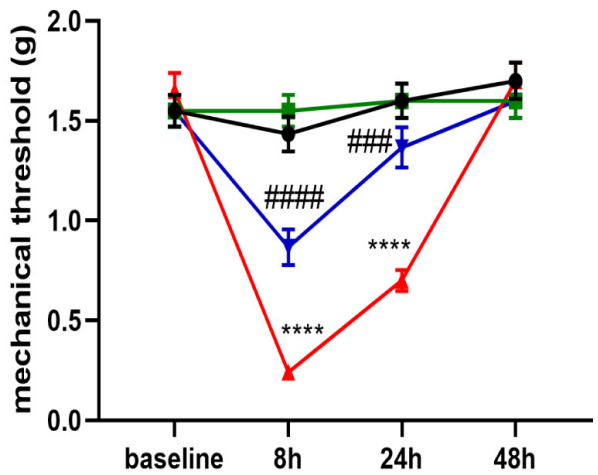

Saline + Saline

$\rightarrow$ DEX + Saline

\pm Saline + LPS

$\rightarrow$ DEX + LPS

FIGURE 8 | DEX pretreatment attenuated LPS-induced mechanical hyperalgesia. (A) Representative photomicrographs of myeloperoxidase (MPO) immunostaining in the rat spinal cord dorsal horn $8 \mathrm{~h}$ after saline or LPS injection. Scale bars, $100 \mu \mathrm{m}$. (B) Systemic LPS exposure did not induce obvious accumulation of MPO-positive cells $8 \mathrm{~h}$ after LPS injection. (C) Experimental design. P5-6 rat pups underwent intraperitoneal injection of saline or DEX. The baseline mechanical threshold was measured $1 \mathrm{~h}$ before saline or DEX injection. Thirty minutes after the injection, the rat pups were intraperitoneally injected with saline or LPS. Rat pups underwent a nociceptive behavior test 8, 24, and $48 \mathrm{~h}$ after LPS injection, or were sacrificed for mRNA analyses and ELISA $8 \mathrm{~h}$ after LPS injection. (D) Mechanical threshold was decreased in rats 8 and $24 \mathrm{~h}$ after LPS injection, and DEX significantly alleviated LPS-induced mechanical hyperalgesia. Results of panel (B) are expressed as the mean \pm SEM, $n=4$ animals for each group, and were analyzed by unpaired $t$-test. Results of panel (D) are expressed as the mean \pm SEM, $n=12$ animals for each group and were analyzed by two-way repeated-measures ANOVA followed by Bonferroni's multiple comparisons test. ${ }^{* \star \star *} P<0.0001$ Saline + LPS group vs. Saline + Saline group. ${ }^{\# \#} P<0.001,{ }^{\# \# \#} P<0.0001$ DEX + LPS group vs. Saline + LPS group.

decreased LPS-induced inflammation, M1 microglial marker expressions, and microglial pro-inflammatory cytokine expressions, which consequentially alleviated LPS-induced mechanical hyperalgesia in neonatal rats.
As a major component of the outer membrane of gramnegative bacteria, LPS can induce pain hypersensitivity in both humans and animals (Guo and Schluesener, 2006; Karshikoff et al., 2015; Wegner et al., 2015). Intracerebral injection of 
LPS in neonatal rats could cause a long-lasting hyperalgesia until adulthood (Wang et al., 2011). In our study, we did find that systemic LPS injection induced an acute mechanical hyperalgesia as early as in neonates. This result was consistent with another study that a single systemic LPS injection elicited acute mechanical hyperalgesia in adult rats (Guo and Schluesener, 2006). Unlike LPS-induced hypersensitivity to mechanical stimulation, we did not find any significant changes in thermal latency after LPS injection, compared with a previous study that reported that LPS injection resulted in a reduction in both mechanical threshold and thermal latency in neonatal rats (Hsieh et al., 2018). In their research, a tailflick test was performed to assess thermal latency, while in our study, we performed a plantar test instead. This difference may be possibly caused by the differences in nociceptive behavior assessment methods.

Systemic LPS injection can elicit inflammation in the CNS, but the accurate mechanism has not been fully elucidated. The interaction of LPS and endogenous immune cells in the CNS and the subsequent release of pro-inflammatory cytokines may contribute to LPS-induced neuroinflammation (Lund et al., 2006; Bowyer et al., 2020). Another possible mechanism is the transition of pro-inflammatory cytokines in circulation to the CNS, which activates endogenous immune cells in the CNS and triggers inflammatory responses (Banks et al., 1991; Pan and Kastin, 2002; Qin et al., 2007; Banks and Erickson, 2010; Swaroop et al., 2016; Bras et al., 2020). Consistent with previous studies (Hsieh et al., 2018, 2020), our findings clearly proved that systemic LPS injection induces the release of pro-inflammatory cytokines in the spinal cord of neonatal rats (Figures 3, 6). Previous studies have reported that LPS exposure could result in persistent inflammation in the CNS, which may influence brain function (Qin et al., 2007; O'loughlin et al., 2017; Gisslen et al., 2019). Moreover, rats injected with LPS during the neonatal period also displayed enhanced pain sensitivity later in life (Boisse et al., 2005; Zouikr et al., 2014, 2015). Thus, it is very possible that LPS can induce persistent inflammation in the spinal cord of neonatal rats which mediates central sensitization and pain hypersensitivity later in life. In our study, although our results showed that LPS exposure induced an enhanced short-term accumulation of spinal pro-inflammatory cytokines, which was resolved after $48 \mathrm{~h}$, seeking a suitable experimental animal disease model should also be taken into consideration.

Spinal microglia play a key role in hyperalgesia, and the mechanism includes microglial polarization toward a pro-inflammatory (M1) phenotype and production of proinflammatory cytokines (Kawasaki et al., 2008; Orihuela et al., 2016; Gong et al., 2017). The pro-inflammatory cytokines, namely, IL- $1 \beta$ and TNF- $\alpha$, can induce central sensitization either by increasing excitatory synaptic transmission or decreasing inhibitory synaptic transmission in the dorsal horn of the spinal cord (Kawasaki et al., 2008), which results in the enhancement of pain signal transmission (Ji et al., 2016). Previous studies have reported that spinal microglial activation was implicated in LPS-induced hyperalgesia (Yoon et al., 2012; Hsieh et al., 2018). In our study, we found LPS-induced upregulations of M1 microglial markers in the spinal cord. As microglial responses to a noxious stimulus also include proliferation and morphological changes, i.e., enlarged cell bodies and shortened processes (Chen et al., 2018), we measured microglial cell number and soma size in the dorsal horn of the spinal cord by using Iba1 immunostaining (Figures 4C-G). Although LPS did not induce significant changes in the number of microglial cells $24 \mathrm{~h}$ after LPS injection, microglia did appear to have larger cell bodies, an implication of cell activation. We also noticed a chronological order between the morphological changes in microglia and the inflammatory profiles in the spinal cord, as the expressions of M1 microglial markers and pro-inflammatory cytokines were elevated $8 \mathrm{~h}$ after LPS injection, while the microglial morphological changes took place $24 \mathrm{~h}$ after LPS injection. This finding is consistent with a prior study that microglial morphological changes were delayed compared with microglial inflammatory cytokine expression after the LPS challenge (Norden et al., 2016).

Dexmedetomidine has been widely used in pediatric clinical practice in recent years, and its anti-inflammatory effects have been increasingly studied (Ning et al., 2017; Feng et al., 2019; Li R. et al., 2020). Consistent with these studies, we found that DEX could alleviate inflammation, both in the serum and the spinal cord, in neonatal rats injected with LPS. As the inhibition of microglial activation and microglial polarization toward the M1 phenotype has contributed to the anti-inflammatory property of DEX in the CNS (Yeh et al., 2018; Gao et al., 2019; Meng et al., 2020), we next examined the effects of DEX on the expression levels of M1 microglial markers and indeed found that DEX pretreatment decreased the LPS-induced expressions of M1 microglial markers in the spinal cord of neonatal rats. To further confirm the protective effects of DEX on microglia, we sorted microglia from the spinal cord and found that DEX pretreatment decreased LPS-induced expressions of proinflammatory cytokines in microglia. Considering that the proinflammatory cytokines in circulation can transport to the CNS, which may activate microglia and trigger inflammatory responses (Banks et al., 1991; Pan and Kastin, 2002; Qin et al., 2007; Banks and Erickson, 2010; Swaroop et al., 2016; Bras et al., 2020), the anti-neuroinflammation effect of DEX in our study might be partially through the alleviation of periphery inflammation as well, which subsequently inhibits microglial activation and microglial polarization toward the M1 phenotype. Meanwhile, microglia can be beneficial (Gomes-Leal, 2012). Microglia can polarize toward the M2 phenotype and release anti-inflammatory cytokines, such as IL-10 and IL-4, under certain conditions (Orihuela et al., 2016). It has been shown that IL-10 and IL-4 could suppress pro-inflammatory responses (Marques et al., 2004). A previous study has demonstrated that blocking IL-10 activity could result in hyperalgesia (Mckelvey et al., 2015). As DEX can induce M2 microglial polarization and increase the levels of IL-10 and IL-4 in microglia (Sun et al., 2018; Gao et al., 2019; Qiu et al., 2020), it is very possible that the upregulation of microglial anti-inflammatory cytokine expressions in the spinal cord may also be implicated in the anti-inflammatory and anti-hyperalgesia effects of DEX. However, in our model, we did not detect a significant difference in the expressions of IL-10 and IL- 4 in microglia $8 \mathrm{~h}$ after 
LPS injection. Moreover, there were no obvious effects of DEX on the expressions of IL-10 and IL- 4 in the microglia. Thus, the effect of anti-inflammation could occur in the late stage after LPS injection.

We also noticed the inconsistency of the pro-inflammatory cytokine mRNA expressions between the sorted microglia and the spinal cord tissue, which indicated the possible involvement of other cells in spinal inflammation. Astrocytes, another type of glial cells in the CNS, have been reported to produce iNOS and CD86 as well as pro-inflammatory cytokines in response to stimulus in vitro (Soos et al., 1999; Cheng et al., 2020). However, astrogliosis and increase in cyclooxygenase-2 (COX-2) have been mainly found in vivo in the spinal cord of neonatal rats after LPS injection (Hsieh et al., 2018, 2020). A detailed study on astrocytic contribution needs to be fully investigated in the future. Neutrophils are important inflammatory cells that are upregulated in the CNS after damage and are a source of pro-inflammatory cytokines (Ji et al., 2007; Steinbach et al., 2013; Alam et al., 2020). It has been shown that neutrophils could be observed in the brain after systemic LPS injection (Zhou et al., 2009; Wu et al., 2020). Microglia can be activated and phagocytize neutrophils (Neumann et al., 2008). Therefore, we detected neutrophils in the dorsal horn of the spinal cord $8 \mathrm{~h}$ after LPS injection, at which time point the inflammation was drastic and DEX showed an obvious anti-inflammatory property. However, there was no obvious accumulation of neutrophils at this time point. More detailed studies on the involvement of neutrophils in LPS-induced spinal inflammation are required in the future.

In summary, we demonstrated that a single systemic LPS injection induced acute mechanical hyperalgesia, systemic inflammation, and short-term pro-inflammatory cytokine production and microglial activation in the spinal cord of neonatal rats. Pretreatment with DEX significantly decreased the spinal inflammation and alleviated the mechanical hyperalgesia induced by LPS, which is possible through the inhibition of M1 microglial polarization and microglial pro-inflammatory

\section{REFERENCES}

Alam, A., Thelin, E. P., Tajsic, T., Khan, D. Z., Khellaf, A., Patani, R., et al. (2020). Cellular infiltration in traumatic brain injury. J. Neuroinflamm. 17:328.

Banks, W. A., and Erickson, M. A. (2010). The blood-brain barrier and immune function and dysfunction. Neurobiol. Dis. 37, 26-32. doi: 10.1016/j.nbd.2009. 07.031

Banks, W. A., Ortiz, L., Plotkin, S. R., and Kastin, A. J. (1991). Human interleukin (IL) 1 alpha, murine IL-1 alpha and murine IL-1 beta are transported from blood to brain in the mouse by a shared saturable mechanism. J. Pharmacol. Exp. Ther. 259, 988-996.

Boisse, L., Spencer, S. J., Mouihate, A., Vergnolle, N., and Pittman, Q. J. (2005). Neonatal immune challenge alters nociception in the adult rat. Pain 119, 133-141. doi: 10.1016/j.pain.2005.0 9.022

Bowyer, J. F., Sarkar, S., Burks, S. M., Hess, J. N., Tolani, S., O'callaghan, J. P., et al. (2020). Microglial activation and responses to vasculature that result from an acute LPS exposure. Neurotoxicology 77, 181-192. doi: 10.1016/j.neuro.2020. 01.014

Bras, J. P., Bravo, J., Freitas, J., Barbosa, M. A., Santos, S. G., Summavielle, T., et al. (2020). TNF-alpha-induced microglia activation requires miR-342: impact cytokine expression. Therefore, our finding highlights a new therapeutic target in the treatment of infection-induced hyperalgesia in neonates and preterm infants. This treatment may further prevent cognitive deficits and abnormal pain sensitivity caused by noxious stimuli and painful experiences in early life.

\section{DATA AVAILABILITY STATEMENT}

The original contributions presented in the study are included in the article/supplementary material, further inquiries can be directed to the corresponding author/s.

\section{ETHICS STATEMENT}

The animal study was reviewed and approved by the Laboratory Animal Welfare and Ethics Committee of Shanghai Children's Medical Center.

\section{AUTHOR CONTRIBUTIONS}

WW, XG, JZ, XT, and MZ designed the study. WW, HC, and YY collected the data. WW, XG, and MC analyzed the data. WW drafted the manuscript. XT and MZ reviewed, edited, and finalized the manuscript. All authors contributed to the article and approved the submitted version.

\section{FUNDING}

This research was supported by grants from the Shanghai Committee of Science and Technology (No. 20ZR1434600); the National Natural Science Foundation of China (No. 81270414); the Shanghai Municipal Commission of Health and Family Planning, and the Key Developing Disciplines (No. 2015ZB0106). on NF-kB signaling and neurotoxicity. Cell Death Dis. 11:415. doi: 10.1038/ s41419-020-2626-2626

Carlos, T. M., Clark, R. S., Franicola-Higgins, D., Schiding, J. K., and Kochanek, P. M. (1997). Expression of endothelial adhesion molecules and recruitment of neutrophils after traumatic brain injury in rats. J. Leukoc. Biol. 61, 279-285. doi: 10.1002/jlb.61.3.279

Chen, G., Zhang, Y. Q., Qadri, Y. J., Serhan, C. N., and Ji, R. R. (2018). Microglia in pain: detrimental and protective roles in pathogenesis and resolution of pain. Neuron 100, 1292-1311. doi: 10.1016/j.neuron.2018.11.009

Chen, J., Suo, S., Tam, P. P., Han, J. J., Peng, G., and Jing, N. (2017). Spatial transcriptomic analysis of cryosectioned tissue samples with Geo-seq. Nat. Protoc. 12, 566-580. doi: 10.1038/nprot.2017.003

Chen, M., Shi, X., Chen, Y., Cao, Z., Cheng, R., Xu, Y., et al. (2012). A prospective study of pain experience in a neonatal intensive care unit of China. Clin. J. Pain 28, 700-704. doi: 10.1097/AJP.0b013e3182400d54

Cheng, Y. Y., Ding, Y. X., Bian, G. L., Chen, L. W., Yao, X. Y., Lin, Y. B., et al. (2020). Reactive astrocytes display pro-inflammatory adaptability with modulation of Notch-PI3K-AKT signaling pathway under inflammatory stimulation. Neuroscience 440, 130-145. doi: 10.1016/j.neuroscience.2020.05.023

Feng, X., Guan, W., Zhao, Y., Wang, C., Song, M., Yao, Y., et al. (2019). Dexmedetomidine ameliorates lipopolysaccharide-induced acute kidney injury 
in rats by inhibiting inflammation and oxidative stress via the GSK-3beta/Nrf2 signaling pathway. J. Cell. Physiol. 234, 18994-19009. doi: 10.1002/jcp.28539

Gao, J., Sun, Z., Xiao, Z., Du, Q., Niu, X., Wang, G., et al. (2019). Dexmedetomidine modulates neuroinflammation and improves outcome via alpha2-adrenergic receptor signaling after rat spinal cord injury. Br. J. Anaesth. 123, 827-838. doi: 10.1016/j.bja.2019.08.026

Gisslen, T., Singh, G., and Georgieff, M. K. (2019). Fetal inflammation is associated with persistent systemic and hippocampal inflammation and dysregulation of hippocampal glutamatergic homeostasis. Pediatr. Res. 85, 703-710. doi: 10. 1038/s41390-019-0330-y

Gomes-Leal, W. (2012). Microglial physiopathology: how to explain the dual role of microglia after acute neural disorders? Brain Behav. 2, 345-356. doi: 10.1002/ brb3.51

Gong, X., Chen, Y., Fu, B., Jiang, J., and Zhang, M. (2017). Infant nerve injury induces delayed microglial polarization to the M1 phenotype, and exercise reduces delayed neuropathic pain by modulating microglial activity. Neuroscience 349, 76-86. doi: 10.1016/j.neuroscience.2017.02.051

Gong, X., Jiang, J., and Zhang, M. (2016). Exercise preconditioning reduces neonatal incision surgery-induced enhanced hyperalgesia via inhibition of $\mathrm{P} 38$ mitogen-activated protein kinase and IL-1beta. TNF-alpha release. Int. J. Dev. Neurosci. 52, 46-54. doi: 10.1016/j.ijdevneu.2016.05.008

Guo, L. H., and Schluesener, H. J. (2006). Acute but not chronic stimulation of glial cells in rat spinal cord by systemic injection of lipopolysaccharide is associated with hyperalgesia. Acta Neuropathol. 112, 703-713. doi: 10.1007/s00401-0060135-z

Hermann, C., Hohmeister, J., Demirakca, S., Zohsel, K., and Flor, H. (2006). Long-term alteration of pain sensitivity in school-aged children with early pain experiences. Pain 125, 278-285. doi: 10.1016/j.pain.2006.08.026

Hsieh, C. T., Lee, Y. J., Dai, X., Ojeda, N. B., Lee, H. J., Tien, L. T., et al. (2018). Systemic Lipopolysaccharide-induced pain sensitivity and spinal inflammation were reduced by minocycline in neonatal rats. Int. J. Mol. Sci. 19:2947. doi: 10.3390/ijms19102947

Hsieh, C. T., Lee, Y. J., Lee, J. W., Lu, S., Tucci, M. A., Dai, X., et al. (2020). Interleukin-1 receptor antagonist ameliorates the pain hypersensitivity, spinal inflammation and oxidative stress induced by systemic lipopolysaccharide in neonatal rats. Neurochem. Int. 135:104686. doi: 10.1016/j.neuint.2020.104686

Huo, W., Zhang, Y., Liu, Y., Lei, Y., Sun, R., Zhang, W., et al. (2018). Dehydrocorydaline attenuates bone cancer pain by shifting microglial M1/M2 polarization toward the M2 phenotype. Mol. Pain 14:1744806918781733. doi: $10.1177 / 1744806918781733$

Ji, K. A., Yang, M. S., Jeong, H. K., Min, K. J., Kang, S. H., Jou, I., et al. (2007). Resident microglia die and infiltrated neutrophils and monocytes become major inflammatory cells in lipopolysaccharide-injected brain. Glia 55, 1577-1588. doi: $10.1002 /$ glia.20571

Ji, R. R., Chamessian, A., and Zhang, Y. Q. (2016). Pain regulation by non-neuronal cells and inflammation. Science 354, 572-577. doi: 10.1126/science.aaf8924

Karshikoff, B., Lekander, M., Soop, A., Lindstedt, F., Ingvar, M., Kosek, E., et al. (2015). Modality and sex differences in pain sensitivity during human endotoxemia. Brain Behav. Immun. 46, 35-43. doi: 10.1016/j.bbi.2014.11.014

Kawasaki, Y., Zhang, L., Cheng, J. K., and Ji, R. R. (2008). Cytokine mechanisms of central sensitization: distinct and overlapping role of interleukin-1beta, interleukin-6, and tumor necrosis factor-alpha in regulating synaptic and neuronal activity in the superficial spinal cord. J. Neurosci. 28, 5189-5194. doi: 10.1523/JNEUROSCI.3338-07.2008

Kong, L. L., Wang, Z. Y., Han, N., Zhuang, X. M., Wang, Z. Z., Li, H., et al. (2014). Neutralization of chemokine-like factor 1 , a novel C-C chemokine, protects against focal cerebral ischemia by inhibiting neutrophil infiltration via MAPK pathways in rats. J. Neuroinflamm. 11:112. doi: 10.1186/1742-2094-11-112

Li, J., Shi, C., Ding, Z., and Jin, W. (2020). Glycogen synthase kinase 3beta promotes postoperative cognitive dysfunction by inducing the M1 polarization and migration of microglia. Med. Inflamm. 2020:7860829. doi: 10.1155/2020/ 7860829

Li, R., Lai, I. K., Pan, J. Z., Zhang, P., and Maze, M. (2020). Dexmedetomidine exerts an anti-inflammatory effect via alpha2 adrenoceptors to prevent Lipopolysaccharide-induced cognitive decline in mice. Anesthesiology 133, 393407. doi: 10.1097/ALN.0000000000003390

Lund, S., Christensen, K. V., Hedtjarn, M., Mortensen, A. L., Hagberg, H., Falsig, J., et al. (2006). The dynamics of the LPS triggered inflammatory response of murine microglia under different culture and in vivo conditions. J. Neuroimmunol. 180, 71-87. doi: 10.1016/j.jneuroim.2006.07.007

Mahmoud, M., Barbi, E., and Mason, K. P. (2020). Dexmedetomidine: what's new for pediatrics? a narrative review. J. Clin. Med. 9:2724. doi: 10.3390/jcm 9092724

Marques, C. P., Hu, S., Sheng, W., Cheeran, M. C., Cox, D., and Lokensgard, J. R. (2004). Interleukin-10 attenuates production of HSV-induced inflammatory mediators by human microglia. Glia 47, 358-366. doi: 10.1002/glia.20045

Mckelvey, R., Berta, T., Old, E., Ji, R. R., and Fitzgerald, M. (2015). Neuropathic pain is constitutively suppressed in early life by anti-inflammatory neuroimmune regulation. J. Neurosci. 35, 457-466. doi: 10.1523/JNEUROSCI. 2315-14.2015

Meng, F., Yu, W., Duan, W., Wang, T., and Liu, Y. (2020). Dexmedetomidine attenuates LPS-mediated BV2 microglia cells inflammation via inhibition of glycolysis. Fundam. Clin. Pharmacol. 34, 313-320. doi: 10.1111/fcp.12528

Neumann, J., Sauerzweig, S., Ronicke, R., Gunzer, F., Dinkel, K., Ullrich, O., et al. (2008). Microglia cells protect neurons by direct engulfment of invading neutrophil granulocytes: a new mechanism of CNS immune privilege. J. Neurosci. 28, 5965-5975. doi: 10.1523/JNEUROSCI.0060-08.2008

Ning, Q., Liu, Z., Wang, X., Zhang, R., Zhang, J., Yang, M., et al. (2017). Neurodegenerative changes and neuroapoptosis induced by systemic lipopolysaccharide administration are reversed by dexmedetomidine treatment in mice. Neurol. Res. 39, 357-366. doi: 10.1080/01616412.2017.128 1197

Norden, D. M., Trojanowski, P. J., Villanueva, E., Navarro, E., and Godbout, J. P. (2016). Sequential activation of microglia and astrocyte cytokine expression precedes increased Iba-1 or GFAP immunoreactivity following systemic immune challenge. Glia 64, 300-316. doi: 10.1002/glia.22930

Nuseir, K. Q., Alzoubi, K. H., Alhusban, A., Bawaane, A., Al-Azzani, M., and Khabour, O. F. (2017). Sucrose and naltrexone prevent increased pain sensitivity and impaired long-term memory induced by repetitive neonatal noxious stimulation: role of BDNF and beta-endorphin. Physiol. Behav. 179, 213-219. doi: 10.1016/j.physbeh.2017.06.015

O’loughlin, E., Pakan, J. M. P., Yilmazer-Hanke, D., and Mcdermott, K. W. (2017). Acute in utero exposure to lipopolysaccharide induces inflammation in the pre- and postnatal brain and alters the glial cytoarchitecture in the developing amygdala. J. Neuroinflamm. 14:212. doi: 10.1186/s12974-0170981-8

Orihuela, R., Mcpherson, C. A., and Harry, G. J. (2016). Microglial M1/M2 polarization and metabolic states. Br. J. Pharmacol. 173, 649-665. doi: 10.1111/ bph.13139

Paeschke, N., Von Haefen, C., Endesfelder, S., Sifringer, M., and Spies, C. D. (2017). Dexmedetomidine prevents Lipopolysaccharide-induced microRNA expression in the adult rat brain. Int. J. Mol. Sci. 18:1830. doi: 10.3390/ ijms18091830

Pan, W., and Kastin, A. J. (2002). TNFalpha transport across the blood-brain barrier is abolished in receptor knockout mice. Exp. Neurol. 174, 193-200. doi: 10.1006/exnr.2002.7871

Qin, L., Wu, X., Block, M. L., Liu, Y., Breese, G. R., Hong, J. S., et al. (2007). Systemic LPS causes chronic neuroinflammation and progressive neurodegeneration. Glia 55, 453-462. doi: 10.1002/glia.20467

Qiu, Z., Lu, P., Wang, K., Zhao, X., Li, Q., Wen, J., et al. (2020). Dexmedetomidine inhibits neuroinflammation by altering microglial M1/M2 polarization through MAPK/ERK pathway. Neurochem. Res. 45, 345-353. doi: 10.1007/s11064-01902922-2921

Ranger, M., Zwicker, J. G., Chau, C. M., Park, M. T., Chakravarthy, M. M., Poskitt, K., et al. (2015). Neonatal pain and infection relate to smaller cerebellum in very preterm children at school age. J. Pediatr. 167:e291. doi: 10.1016/j.jpeds.2015. 04.055

Rudd, K. E., Johnson, S. C., Agesa, K. M., Shackelford, K. A., Tsoi, D., Kievlan, D. R., et al. (2020). Global, regional, and national sepsis incidence and mortality, 19902017: analysis for the Global Burden of Disease Study. Lancet 395, 200-211. doi: 10.1016/s0140-6736(19)32989-32987

Soos, J. M., Ashley, T. A., Morrow, J., Patarroyo, J. C., Szente, B. E., and Zamvil, S. S. (1999). Differential expression of B7 co-stimulatory molecules by astrocytes correlates with $\mathrm{T}$ cell activation and cytokine production. Int. Immunol. 11, 1169-1179. doi: 10.1093/intimm/11.7.1169

Steinbach, K., Piedavent, M., Bauer, S., Neumann, J. T., and Friese, M. A. (2013). Neutrophils amplify autoimmune central nervous system infiltrates by 
maturing local APCs. J. Immunol. 191, 4531-4539. doi: 10.4049/jimmunol. 1202613

Sun, Z., Lin, Y., Li, Y., Ren, T., Du, G., Wang, J., et al. (2018). The effect of dexmedetomidine on inflammatory inhibition and microglial polarization in BV-2 cells. Neurol. Res. 40, 838-846. doi: 10.1080/01616412.2018.1493849

Swaroop, S., Sengupta, N., Suryawanshi, A. R., Adlakha, Y. K., and Basu, A. (2016). HSP60 plays a regulatory role in IL-1beta-induced microglial inflammation via TLR4-p38 MAPK axis. J. Neuroinflamm. 13:27. doi: 10.1186/s12974-0160486-x

Wang, K. C., Wang, S. J., Fan, L. W., Cai, Z., Rhodes, P. G., and Tien, L. T. (2011). Interleukin-1 receptor antagonist ameliorates neonatal lipopolysaccharideinduced long-lasting hyperalgesia in the adult rats. Toxicology 279, 123-129. doi: 10.1016/j.tox.2010.10.002

Wegner, A., Elsenbruch, S., Maluck, J., Grigoleit, J. S., Engler, H., Jager, M., et al. (2014). Inflammation-induced hyperalgesia: effects of timing, dosage, and negative affect on somatic pain sensitivity in human experimental endotoxemia. Brain Behav. Immun. 41, 46-54. doi: 10.1016/j.bbi.2014.05.001

Wegner, A., Elsenbruch, S., Rebernik, L., Roderigo, T., Engelbrecht, E., Jager, M., et al. (2015). Inflammation-induced pain sensitization in men and women: does sex matter in experimental endotoxemia? Pain 156, 1954-1964. doi: 10.1097/j. pain.0000000000000256

Wu, F., Chen, X., Zhai, L., Wang, H., Sun, M., Song, C., et al. (2020). CXCR2 antagonist attenuates neutrophil transmigration into brain in a murine model of LPS induced neuroinflammation. Biochem. Biophys. Res. Commun. 529, 839-845. doi: 10.1016/j.bbrc.2020.05.124

Yeh, C. H., Hsieh, L. P., Lin, M. C., Wei, T. S., Lin, H. C., Chang, C. C., et al. (2018). Dexmedetomidine reduces lipopolysaccharide induced neuroinflammation, sickness behavior, and anhedonia. PLoS One 13:e0191070. doi: 10.1371/journal. pone.0191070

Yoon, S. Y., Patel, D., and Dougherty, P. M. (2012). Minocycline blocks lipopolysaccharide induced hyperalgesia by suppression of microglia but not astrocytes. Neuroscience 221, 214-224. doi: 10.1016/j.neuroscience.2012. 06.024
Zhang, G. X., Li, J., Ventura, E., and Rostami, A. (2002). Parenchymal microglia of naive adult C57BL/6J mice express high levels of B7.1. B7.2, and MHC class II. Exp. Mol. Pathol. 73, 35-45. doi: 10.1006/exmp.2002.2441

Zhou, H., Andonegui, G., Wong, C. H., and Kubes, P. (2009). Role of endothelial TLR4 for neutrophil recruitment into central nervous system microvessels in systemic inflammation. J. Immunol. 183, 5244-5250. doi: 10.4049/jimmunol. 0901309

Zouikr, I., Ahmed, A. F., Horvat, J. C., Beagley, K. W., Clifton, V. L., Ray, A., et al. (2015). Programming of formalin-induced nociception by neonatal LPS exposure: maintenance by peripheral and central neuroimmune activity. Brain Behav. Immun. 44, 235-246. doi: 10.1016/j.bbi.2014.10.014

Zouikr, I., Tadros, M. A., Barouei, J., Beagley, K. W., Clifton, V. L., Callister, R. J., et al. (2014). Altered nociceptive, endocrine, and dorsal horn neuron responses in rats following a neonatal immune challenge. Psychoneuroendocrinology 41, 1-12. doi: 10.1016/j.psyneuen.2013.11.016

Conflict of Interest: The authors declare that the research was conducted in the absence of any commercial or financial relationships that could be construed as a potential conflict of interest.

Publisher's Note: All claims expressed in this article are solely those of the authors and do not necessarily represent those of their affiliated organizations, or those of the publisher, the editors and the reviewers. Any product that may be evaluated in this article, or claim that may be made by its manufacturer, is not guaranteed or endorsed by the publisher.

Copyright (c) 2021 Wen, Gong, Cheung, Yang, Cai, Zheng, Tong and Zhang. This is an open-access article distributed under the terms of the Creative Commons Attribution License (CC BY). The use, distribution or reproduction in other forums is permitted, provided the original author(s) and the copyright owner(s) are credited and that the original publication in this journal is cited, in accordance with accepted academic practice. No use, distribution or reproduction is permitted which does not comply with these terms. 